\title{
Abnormal activation of the synuclein-gamma gene in hepatocellular carcinomas by epigenetic alteration
}

\author{
WEI ZHAO $^{1 *}$, HAIYAN LIU $^{2 *}$, WEI LIU ${ }^{*}$, YINWEI WU ${ }^{1}$, WEI CHEN ${ }^{2}$, BIN JIANG $^{3}$, \\ YUE ZHOU ${ }^{2}$, RONG XUE ${ }^{1}$, CHAN LUO $^{1}$, LAN WANG $^{1}$, JIAN-DONG JIANG ${ }^{4}$ and JINGWEN LIU ${ }^{2}$ \\ ${ }^{1}$ Second Hospital of Nanjing City, Nanjing, P.R. China; ${ }^{2}$ Department of Veterans Affairs \\ Palo Alto Health Care System, Palo Alto, CA, USA; ${ }^{3}$ First Hospital of Nanjing City, Nanjing; \\ ${ }^{4}$ Institute of Medicinal Biotechnology, Chinese Academy of Medicinal Sciences, Beijing, P.R. China
}

Received November 7, 2005; Accepted December 13, 2005

\begin{abstract}
Liver cancer is the fifth most common neoplastic disease and the fourth leading cause of cancer-related death. Identification of the key molecular targets involved in hepatocarcinogenesis has significant therapeutic implications. In this study, by conducting immunohistochemistry, we show that the neuronal protein, synuclein-gamma (SNCG), is abnormally expressed in a high percentage of liver cancer (46/70, 65.7\%). The expression of SNCG in liver cancer exhibits a clear stagespecific pattern of low expression in stage I $(1 / 19,5.3 \%)$ and high expression in stages III to IV $(44 / 50,88 \%)$. Importantly, all patients with metastatic diseases expressed SNCG in their primary tumors $(15 / 15,100 \%)$. Consistent with the IHC results, RT-PCR assays demonstrate that SNCG mRNA is highly expressed in the tumor tissue of advanced hepatocellular carcinomas. Analyses of the methylation status of the $\mathrm{CpG}$ island of the SNCG gene by methylation-specific PCR confirmed that all tumor samples contained the demethylated gene. To determine whether demethylation of SNCG is an early event of genetic abnormality in the process of hepatocarcinogenesis, we examined the methylation status of SNCG in 70 non-malignant cirrhotic liver samples and showed that $64.3 \%$ cirrhotic liver samples contained the partially or completely demethylated gene. We further show that SNCG expression in liver cancer is not restricted to HBV- and HCVinfected tumors, implying the involvement of other hepatocarcinogenic risk factors in SNCG gene reactivation. Utilizing human hepatoma-derived cell line HepG2 as an in vitro model, we demonstrate that hepatic carcinogens aflatoxin B1 and N-
\end{abstract}

Correspondence to: Dr Jingwen Liu (154P), VA Palo Alto Health Care System, 3801 Miranda Avenue, Palo Alto, CA 94304, USA

E-mail: jingwen.liu@med.va.gov

${ }^{*}$ Contributed equally

Key words: synuclein-gamma, DNA methylation, liver cancer, liver cirrhosis, metastasis nitrosodimethylamine (DMN) are strong inducers of SNCG expression. Collectively, these new findings suggest that SNCG protein expression in primary tumors is a strong indicator of distant metastasis and demethylation of SNCG CpG island is an early sign of genetic abnormality in liver cirrhosis preceding hepatocarcinogenesis. Our studies also suggest that inducing demethylation of SNCG by hepatocarcinogens may represent one underlying mechanism for the aberrant expression of SNCG in HCC.

\section{Introduction}

Primary liver cancer is one of the most frequent neoplastic diseases with the majority being hepatocellular carcinoma (HCC) $(1,2)$. It is estimated that over 500,000 new cases of HCC arise per year worldwide and approximately 18,000 new cases of HCC are projected to occur in the United States in 2005 (3). Due to difficulties in early diagnosis and the nature of rapid disease progression, the majority of patients present with advanced stages of HCC. Thus, the prognosis of $\mathrm{HCC}$ remains poor, despite the many treatment options.

Approximately $60-80 \%$ of HCC arise from cirrhotic diseases. Chronic hepatitis $\mathrm{B}$ and $\mathrm{C}$ virus infection, environmental carcinogen exposure, and alcohol abuse are causative factors in liver cirrhosis and are well-recognized prominent risk factors in the development of liver cancer $(3,4)$. However, the underlying molecular mechanisms for the malignant transformation from non-cancerous cirrhosis to HCC remain largely unknown.

Abnormalities in hepatocyte growth factor expression $(5,6)$, extracellular matrix protein depositions $(7,8)$, cell signaling pathways (9), mutations of tumor suppressor genes (10), and activation of oncogenes (11) are observed in both chronic and malignant liver diseases. Recently, epigenetic changes through alternation of the methylation status of $\mathrm{CpG}$ islands of cellular genes have become an increasingly recognized contributing factor in cancer development. Hypermethylations of tumor suppressor genes are frequently detected in liver cancer $(2,12-14)$.

The human synuclein-gamma (SNCG) gene (15), also named persyn (16) or BCSG1 (breast cancer specific gene 1) (17), is a tissue-specific gene primarily expressed in brain tissue and is abundant in presynaptic terminals. The exon 1 
region of the SNCG gene contains a CpG island (18). Fifteen $\mathrm{CpG}$ dinucleotides within the island are heavily methylated in tissues outside the neuronal system, resulting in the transcriptional inert (19). However, during malignant transformation, the $\mathrm{CpG}$ island of the SNCG gene became demethylated and led to the aberrant expression of this neuronal protein in cancer cells (18-20). The loss of methylation control of the SNCG gene was first discovered in breast carcinomas in which SNCG is abundantly expressed in a stage-specific fashion. While SNCG is not expressed in normal or benign breast tissues, it is highly expressed in advanced and metastatic breast carcinomas $(17,21,22)$. When overexpressed, SNCG induces cell proliferation (23-26), increases cell motility (27), augments tumor cell invasion and metastasis (27), and enhances the resistance of tumor cells to anti-microtubule drug-induced apoptosis (28-30). Thus, in breast cancer cells SNCG behaves as an oncogene.

In a previous pilot study to determine whether SNCG could contribute to liver carcinogenesis, we examined SNCG protein expression and gene demethylation in 20 liver tumor samples of advanced stages and their matched non-neoplastic adjacent tissue (NNAT) (19). SNCG protein was detected in 18/20 tumors and 0/20 NNAT samples. Interestingly, we found that all tumor samples contained a demethylated $\mathrm{CpG}$ island which, in a substantial number of NNAT samples, became demethylated, implying that the demethylation event of the SNCG CpG island precedes malignant transformation in tumor neighboring cells.

To firmly establish the role of SNCG in the disease progression and metastasis of liver cancer and to determine whether SNCG is involved in the malignant conversion of cirrhotic diseases to HCC, we examined the protein expression and methylation status of the SNCG CpG island in primary liver cancer of different histological stages and in non-cancerous liver cirrhotic samples. We further evaluated the associations between SNCG expression and hepatitis virus infection as well as hepatocarcinogen exposure to gain some mechanistic insight into the reawakening of this silent proto oncogene in malignant liver cells.

\section{Materials and methods}

Tissue specimens. With Institutional Review Board approval, 70 paraffin-embedded formalin-fixed blocks of nodular cirrhotic liver tissue and 70 paraffin-embedded formalinfixed blocks of primary liver tumor tissue were obtained from the Second Hospital of Nanjing City, Nanjing, China, and were $H \& E$ counterstained. In addition, 16 pairs of frozen HCC tissue samples and matched non-neoplastic adjacent tissue were obtained from the First Hospital of Nanjing City, Nanjing, China. Tumors were staged following standard American Joint Committee on Carcinoma/International Union Against Carcinoma tumor-node-metastasis (TNM) methodology.

Cells and reagents. Human hepatoma cell line HepG2 was obtained from American Type Culture Collection and cultured in EMEM with $10 \%$ fetal bovine serum (FBS). 5-aza-2'deoxycytidine (5-aza-C), aflatoxin B1 (AFB1), and N-nitrosodimethylamine (DMN) were purchased from Sigma.
Table I. SNCG protein expression status in different types of liver cancer.

\begin{tabular}{lccc}
\hline & & \multicolumn{2}{c}{ SNCG protein expression } \\
\cline { 3 - 4 } Cancer type & Sample no. & No. & \% positivity \\
\hline HCC & 63 & 43 & 68.3 \\
CC & 1 & 1 & 100 \\
MHC & 1 & 0 & 0 \\
MLC & 5 & 2 & 40 \\
$\Sigma$ & 70 & 46 & 65.7 \\
\hline
\end{tabular}

aProtein expression assessed by immunohistochemistry. HCC, hepatocellular carcinoma; CC, cholangiocarcinoma; MHC, mixed hepatocellular cholangiocarcinoma; MLC, metastatic liver cancer.

Immunohistochemistry. Tissue sections of $4 \mu \mathrm{m}$ were deparaffinized with xylene. Following antigen retrieval and blocking of endogenous peroxidase activity (19), slides were incubated with goat anti-SNCG polyclonal antibody (sc-10699, Santa Cruz Biotechnology, Santa Cruz, CA) at 1:300 dilution at $4^{\circ} \mathrm{C}$ overnight. Following 3 washes with PBS, slides were incubated with biotin-labeled rabbit anti-goat IgG (HistostainPlus Kit, 50-232, Zymed) for $30 \mathrm{~min}$ at $37^{\circ} \mathrm{C}$. After 3 washes of PBS, the staining was accomplished by using DAB + Substrate Chromogen Systems (Dako Corp., Carpinteria, CA). Sections were counter-stained with hematoxylin, dehydrated, and mounted. Positive cases were defined by the presence of intracellular staining with red/brown color in malignant cells, as seen in positive controls (paraffin sections from stage III breast cancer patients) (21). Negative cases were defined by the absence of specific intracellular staining, as seen in negative controls consisting of paraffin sections from normal liver tissue. Samples were evaluated under light microscopy independently by two pathologists without prior knowledge of the patients' clinical data.

Similar procedures of IHC and grading system were employed in the detection of HBV surface antigen (HBsAg), $\mathrm{HBV}$ core antigen $(\mathrm{HBcAg})$, and $\mathrm{HCV}$ antigen ( $\mathrm{HCVAg}$ ) in the liver sections of the same patient cohort. Antibodies against HBV surface antigen (HBsAb, ZM-0122), HBV core antigen (HBcAb, RAB-0090), and HCV antigen (HCVAb, ZM-0124) were purchased from Zhangshan Biotechnology Co., Ltd., China.

Nested methylation-specific PCR (NMSP). For each patient sample, genomic DNA was isolated from fifteen $10-\mu \mathrm{m}$ consecutive sections of formalin-fixed, paraffin embedded tissue block as previously described (19). Genomic DNA was resuspended in $30 \mu \mathrm{l}$ of Tris-EDTA buffer and $15 \mu \mathrm{l}$ of DNA was diluted by distilled water to a volume of $50 \mu 1$ and was denatured by $\mathrm{NaOH}$ for $15 \mathrm{~min}$ at $37^{\circ} \mathrm{C}$, followed by treatment with sodium bisulfite at $50^{\circ} \mathrm{C}$ for $16 \mathrm{~h}$ (31). After column purification, the modified DNA was used for NMSP. The sequences of primers used in this study and the PCR conditions have been previously described (19). 

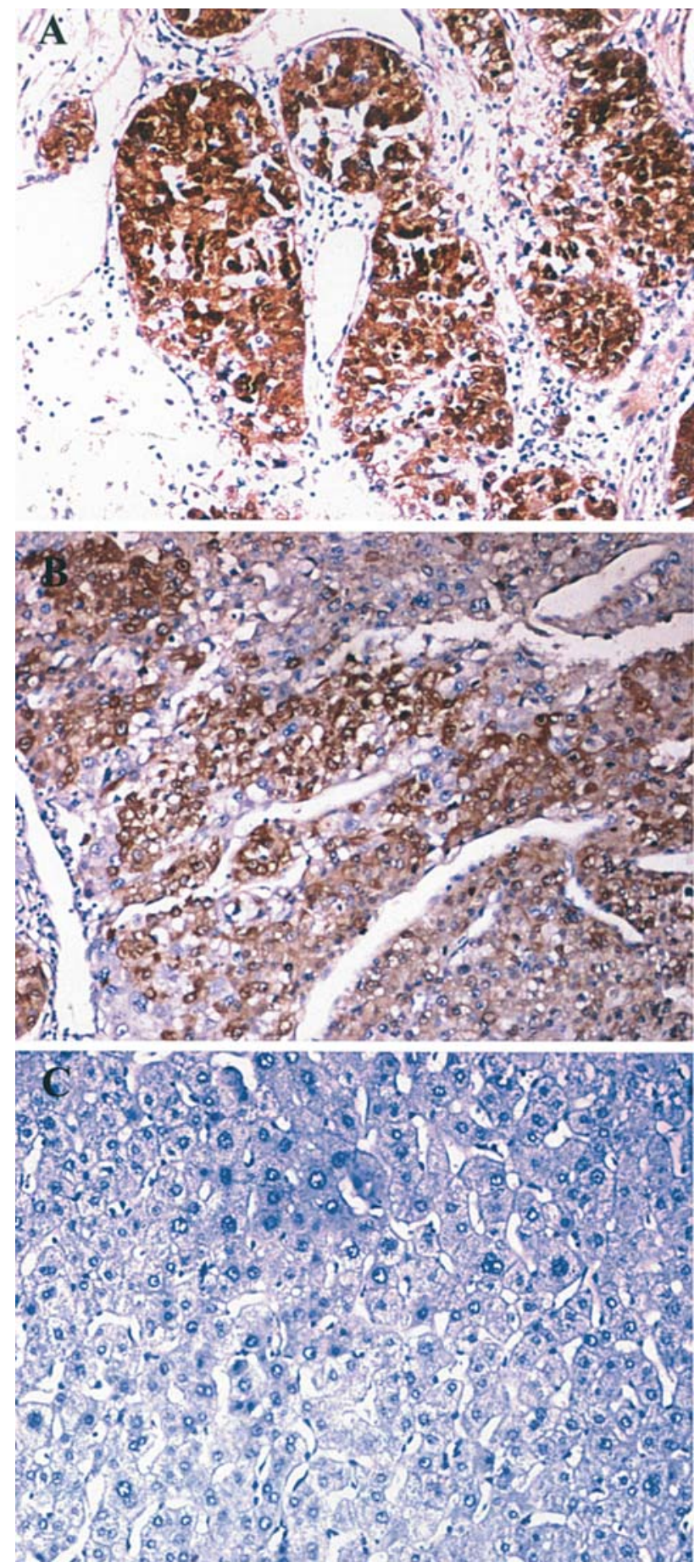

Figure 1. Representative immunohistochemical staining of SNCG protein in liver tumors and normal liver tissue. For each sample, numbers of SNCG positive cells were counted from 5 randomly chosen fields of 400x and averaged. The mean value $(\mathrm{N})$ was used to grade the expression levels: + , $0<\mathrm{N} \leq 30 ;++, 30<\mathrm{N} \leq 50 ;+++, 50<\mathrm{N} \leq 80$. (A) Positive staining at a level of +++ of HCC. (B) Positive staining at a level of ++ of HCC. (C) Negative staining of normal liver tissue.

Isolation of RNA from liver tissues and RT-PCR analysis of SNCG $m R N A$ expression. Primary liver tumor tissues and matched NNAT samples were obtained after surgical resection and stored frozen at $-80^{\circ} \mathrm{C}$. Approximately $100 \mathrm{mg}$ of liver tissue was homogenized in $3 \mathrm{ml}$ of Ultraspec RNA lysis solution (Biotecx Laboratory, Houston, TX) and total RNA was isolated according to the vendor's protocol. For analyzing
SNCG mRNA expression by RT-PCR, $2 \mu \mathrm{g}$ of total RNA was used. The reverse transcription was conducted with Anchored Oligo (dT)20 primer (Invitrogen) using M-MLV Reverse Transcriptase (Promega). The PCR reaction was carried out at $94^{\circ} \mathrm{C}$ for $30 \mathrm{sec}, 60^{\circ} \mathrm{C}$ for $30 \mathrm{sec}$, and $72^{\circ} \mathrm{C}$ for $30 \mathrm{sec}$ with initial activation of the enzyme at $94^{\circ} \mathrm{C}$ for $1.5 \mathrm{~min}$. Thirty cycles were performed for SNCG and GAPDH. The PCR was performed using primers SNCG-RT 5' and SNCGRT 3 ' for SNCG and primers GAPDH-RT 5' and GAPDHRT 3' for GAPDH (18).

Reactivation of SNCG gene expression by DNA demthylation in HepG 2 cells. HepG 2 cells cultured in $10 \%$ FBS were treated with 5-aza-C, AFB1, or DMN at the indicated doses and time points. At the end of treatment, genomic DNA was isolated from untreated or treated cells and $2 \mu \mathrm{g}$ DNA from each sample was modified by sodium bisulfite and subjected to MSP and DNA sequencing. For genomic sequencing, PCR using the primer, SNCG-S5F and SNCG-S5R, was conducted for 30 cycles with an annealing temperature of $60^{\circ} \mathrm{C}$. The 361-bp PCR product covering the region -232 to +129 was gel-purified and ligated into pCR2.1-TOPO cloning vector (Invitrogen). After transformation, plasmid DNA was isolated from individual colonies and subjected to sequencing using M13 as sequencing primer to obtain the entire map of the $S N C G \mathrm{CpG}$ island. RT-PCR was conducted as described for tissue samples.

Statistical analysis. Fisher's exact test was performed to evaluate the difference between SNCG positive and negative groups with relation to histological stages, lymph node invasion, distant metastasis, $\mathrm{HBV}$, and $\mathrm{HCV}$ infection. $\mathrm{P}<0.05$ was considered statistically significant different.

\section{Results}

Detection of SNCG protein and mRNA expression in liver cancer and correlation of SNCG expression with disease progression and distant metastasis. To firmly determine the involvement of SNCG in liver carcinogenesis, we first examined SNCG expression in liver tumors. Archived tumor tissues from 70 patients diagnosed with different types of liver cancer were examined for SNCG protein expression by immunohistochemistry (IHC). Table I summarizes the tumor types and the SNCG expression status. Ninety percent of the liver cancer from this patient cohort were HCC (63/70). Positive staining of the SNCG protein with different intensities was detected exclusively in the cytoplasma of tumor cells (Fig. 1A and B). There was no SNCG staining in normal liver tissue (Fig. 1C). Of a total of 70 tumor samples, $46(65.7 \%)$ were positive and $24(34.2 \%)$ were negative for SNCG immunohistochemical staining. To corroborate the finding of IHC, using RT-PCR assays, we separately examined the SNCG mRNA expression in 16 frozen tissue samples of liver tumors, all of which were diagnosed as HCC with histological grades of II and above. The expression level of SNCG mRNA in the tumor's non-neoplastic adjacent tissue (NNAT) from each patient was also examined. The results demonstrate that, of 16 patient samples, 14 displayed high SNCG mRNA expression in tumor samples $(87.5 \%)$. SNCG mRNA was not 

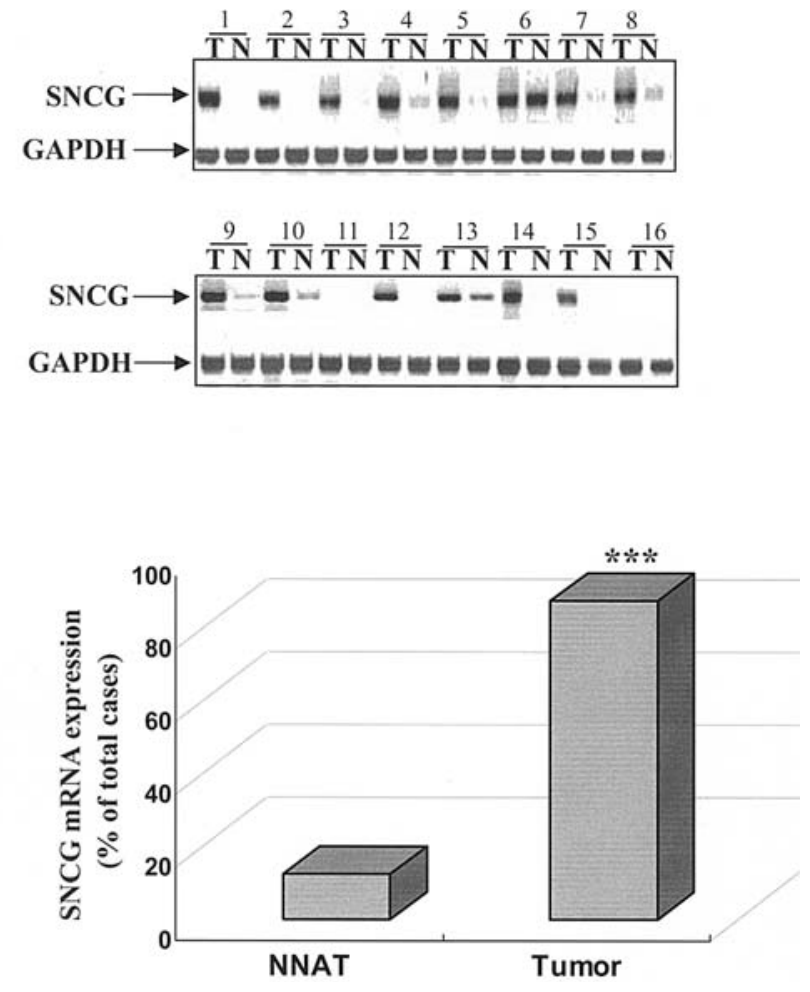

Figure 2. Detection of SNCG mRNA and GAPDH mRNA in primary liver tumors and in matched normal tissue adjacent to the tumors. RNA was isolated from 16 pairs of primary liver tissue where T represents tumor tissue and $\mathrm{N}$ represents non-neoplastic tissue adjacent to the tumor. Expression of SNCG mRNA was analyzed by RT-PCR. The PCR products were visualized on $2 \%$ agarose gels stained with ethidium bromide (upper panel). The bottom bar graph compares the \% positive cases in tumors vs. normal tissue. The expression of GAPDH mRNA was taken as an internal loading control. ${ }^{* * * *} \mathrm{p}<0.001$ compared to normal tissue.

detected in the 12 NNAT samples, whereas trace amounts were shown in the other 2 NNAT samples, and notable expression was found only in N6 and N13 (12.5\%) (p<0.001, Fig. 2). The independent results of IHC and RT-PCR are in good agreement and clearly demonstrate that SNCG is abnormally expressed in a high percentage of liver tumors.

We next evaluated the relationship between the SNCG expression and clinicopathologic factors of the patient cohort (Table II). SNCG protein expression displays a strong correlation with disease progression. Of 19 patients of stage I, only 1 showed a low level of expression $(5.3 \%)$. One patient from this cohort was diagnosed as stage II of HCC and was SNCGpositive. Twenty-nine of 35 stage III patients expressed SNCG (82.9\%). Strikingly, all 15 stage IV metastatic patients expressed SNCG in their primary tumors $(100 \%)$. In addition to the correlation with distant metastasis, SNCG protein expression was also significantly associated with local lymph node invasion. Out of 50 node-positive patients, 44 tumors showed SNCG expression (88\%). These data from a larger size of study group corroborate our previous findings obtained from the pilot study of liver cancer (19) and strongly support our initial hypothesis derived from cell culture studies that SNCG expression stimulates tumor cell invasion and metastasis (26).

Dominant loss of the epigenetic control of the SNCG gene by demethylation of the $C p G$ island in liver cancer. Previously, we
Table II. Correlation between the SNCG protein expression and clinicopathological factors of liver cancer patients.

\begin{tabular}{|c|c|c|c|c|}
\hline \multirow{2}{*}{$\begin{array}{l}\text { Clinicopathological } \\
\text { factors }\end{array}$} & \multirow{2}{*}{$\begin{array}{l}\text { No. of } \\
\text { patients }\end{array}$} & \multicolumn{2}{|c|}{$\begin{array}{l}\text { SNCG protein } \\
\text { expression }\end{array}$} & \\
\hline & & Negative & Positive (\%) & \\
\hline \multicolumn{5}{|l|}{$\operatorname{Sex}$} \\
\hline Male & 60 & 19 & 41 & \\
\hline Female & 10 & 5 & 5 & \\
\hline Age (years) & & $49.0 \pm 13.7$ & $49.9 \pm 12.4$ & \\
\hline \multicolumn{5}{|l|}{ Stage } \\
\hline I & 19 & 18 & 1 & \\
\hline II & 1 & 0 & 1 & \\
\hline III & 35 & 6 & 29 & \\
\hline IV & 15 & 0 & 15 & \\
\hline \multicolumn{5}{|l|}{$\begin{array}{l}\text { Lymph node } \\
\text { invasion }\end{array}$} \\
\hline Positive & 50 & 6 & 44 & \multirow[t]{2}{*}{$\mathrm{p}<0.0001$} \\
\hline Negative & 20 & 18 & 2 & \\
\hline \multicolumn{5}{|l|}{ Distant metastasis } \\
\hline Positive & 15 & 0 & 15 & \multirow{2}{*}{$\mathrm{p}<0.002$} \\
\hline Negative & 55 & 24 & 31 & \\
\hline
\end{tabular}

had developed a highly sensitive method of nested methylationspecific PCR (NMSP) to assess the methylation status of the SNCG CpG island (19). The specificity of this method to faithfully identify unmethylated allele from methylated allele has been approved by DNA sequencing of the PCR products. In this study, to correlate the epigenetic change of the SNCG gene with its aberrant expression in liver cancer, we independently performed NMSP to determine the methylation status of the SNCG CpG island in each tumor sample from the same patient cohort. The upper panel of Fig. 3 shows representative results of 10 DNA samples and all results are summarized in the bar graph of Fig. 3. The demethylated PCR product of the SNCG CpG island was detected in 69/70 tumor samples. In 51 tumor samples, both alleles became completely demethylated and were designated a scale of 2. Eighteen tumor samples contained both methylated and unmethylated alleles, indicating partial demethylation and a scale of 1 . The one tumor sample without demethylation, a scale of 0 , was also negative in SNCG immunostaining. Furthermore, $84.8 \%$ of SNCG-positive tumors contain the fully demethylated gene and only $50 \%$ of SNCGnegative tumors displayed complete demethylation of the SNCG gene. These results indicate that complete demethylation of the $\mathrm{CpG}$ island is probaby required for obtaining high levels of gene transcription to produce enough SNCG protein to be detectable by IHC. Similar to the results of IHC, the extent of demethylation of the SNCG CpG island among tumor samples also significantly correlated with the histological stages of tumors (Fig. 4). The majority of stage III (77.1\%) and stage IV $(86.7 \%)$ patients contain the fully demethylated gene.

To further attest the role of DNA methylation in the control of SNCG gene expression in liver cancer cells, we treated the 

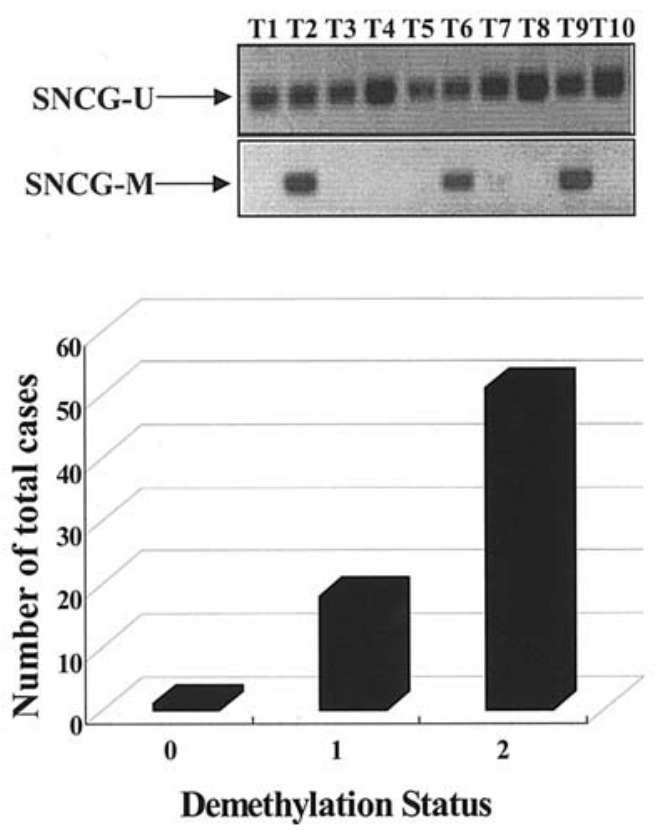

Figure 3. Determination of the SNCG CpG island methylation status in liver tumors by NMSP. NMSP was used to assess the methylation status of SNCG CpG islands in liver tumors. Representative results of 10 tumor samples are shown (upper panel). SNCG-U, unmethylated PCR product; SNCG-M, methylated PCR product. The DNA sample showing only the unmethylated band is scored as 2, the DNA sample showing both unmethylated and methylated is scored 1, the DNA sample without an unmethylated band is designated a score of 0 . The bar graph summarizes the total cases of patient samples that are fully demethylated, partially demethylated, or remaining methylated.

human hepatoma-derived cell line HepG2 cells with the demethylating agent, 5-aza-2'-deoxycytidine (5-aza-C). HepG2 cells predominantly contain the methylated SNCG gene and the expression of SNCG mRNA is barely detectable. MSP indicates that treatment of HepG2 cells with 5-aza-C at a dose of $5 \mu \mathrm{M}$ induced a time-dependent demethylation of the SNCG $\mathrm{CpG}$ island. By the 5th day of treatment, the SNCG gene became fully demethylated (Fig. 5A). We further corroborated the results of MSP with direct genomic sequencing. Fig. 5B shows that $14 / 15 \mathrm{CpG}$ dinucleotides were methylated in HepG2 cells prior to 5 -aza-C treatment and were all converted to TpG after the treatment, indicating the loss of the methyl group at the cytosine residue. Demethylation of the $\mathrm{CpG}$ island was closely accompanied by the expression of SNCG mRNA as detected by RT-PCR assays (Fig. 5C). Collectively, these results clearly demonstrate that loss of the methylation control at the $\mathrm{CpG}$ island is primarily responsible for the abnormally high expression of SNCG in liver tumors.

Aberrant demethylation event in non-neoplastic cirrhotic livers. In our previous pilot studies of 20 pairs of liver tumor and NNAT samples, while SNCG protein was not detected in NNAT samples, demethylated alleles were detected in 9/20 samples of NNAT. Because liver tumors arise largely from the setting of cirrhosis, this prior observation raised an interesting question of whether the demethylation event actually occurs in liver cirrhosis in premalignant stages of liver cancer. To examine this issue, genomic DNA was isolated from 70 paraffin-embedded formalin-fixed blocks of patient samples

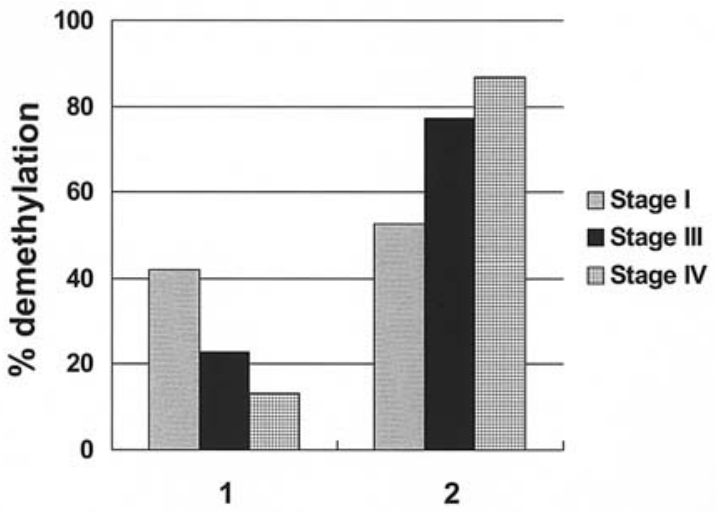

Figure 4. Correlation of different demethylation extents with histological features of liver cancer patients. The DNA sample showing only unmethylated PCR product is scored as 2, the DNA sample showing both unmethylated and methylated PCR products is scored as 1, and the DNA sample showing only methylated PCR products is scored as 0 .

diagnosed as liver nodular cirrhosis. The methylation status of the CpG island in these samples was examined by NMSP. The upper panel of Fig. 6 shows representative results of 10 DNA samples and all results are summarized in the bar graph of Fig. 6. Of the 70 samples, demethylated alleles were readily detected in 45 patients $(64.3 \%)$. Complete demethylation of both alleles was found in 7 samples, while most patients contained the partially demethylated gene. These results demonstrate that the aberrant demethylation event occurs in a substantial population of non-malignant cirrhotic liver diseases and the extent of demethylation is less severe as that in liver tumors.

Aflatoxin B1 exposure reactivates SNCG gene transcription in HepG2 cells. We were interested in identifying cellular and environmental factors that may induce SNCG expression through DNA demethylation, consequently contributing to the onset and progression of liver cancer. Chronic infections of hepatitis B virus (HBV) and hepatitis $\mathrm{C}$ virus (HCV) are among the major risk factors of liver cancer (3). To determine whether $\mathrm{HBV} / \mathrm{HCV}$ infection could be the causative factor for the abnormal expression of SNCG in HCC, we conducted IHC staining of HBV surface and core antigen ( $\mathrm{HBsAg}$, $\mathrm{HBcAg}$ ) and $\mathrm{HCV}$ antigen (HCVAg) on sections from the 70 liver tumors. The results are summarized in Table III. There is no significant correlation between hepatitis virus antigen expression and SNCG expression, indicating that the reactivation of SNCG transcription is independent of hepatitis virus infections.

We next examined the effects of 2 genotoxic hepatocarcinogens, aflatoxin B1 (AFB1) and dimethylnitrosamine (DMN), on the methylation status of the SNCG CpG island. HepG2 cells were treated with non-cytotoxic doses of AFB1 $(1 \mu \mathrm{M})$ or DMN $(100 \mu \mathrm{M})(32)$ for 1 and 3 days. Genomic DNA was subsequently isolated from untreated and treated cells, modified by sodium bisulfite, and subjected to MSP analysis. Fig. 7A shows that the unmethylated PCR product was not present and only methylated PCR product was detected in untreated HepG2 cells. Exposure to hepatocarcinogens rapidly changed the methylation status of the SNCG CpG 
A

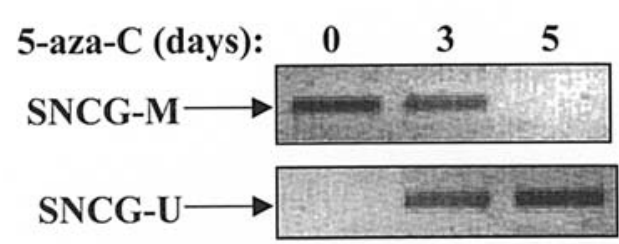

C

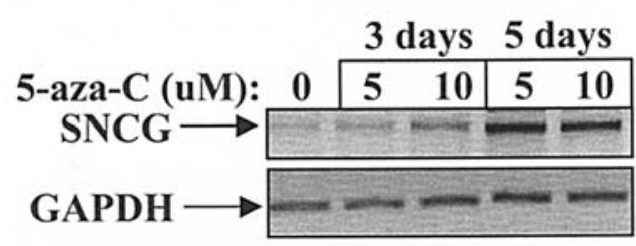

B

SNCG CpG island in HepG2 cell line before 5-aza-C treatment

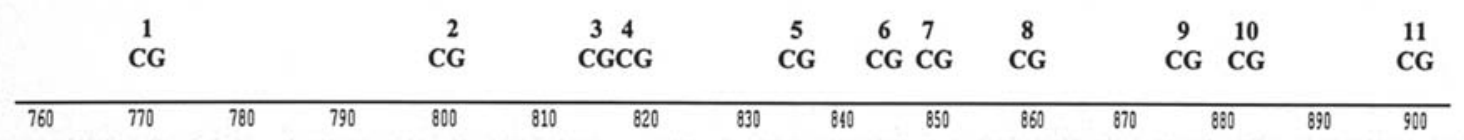

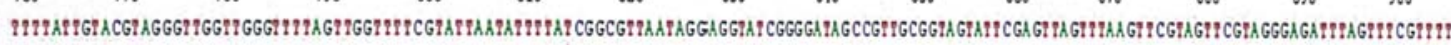

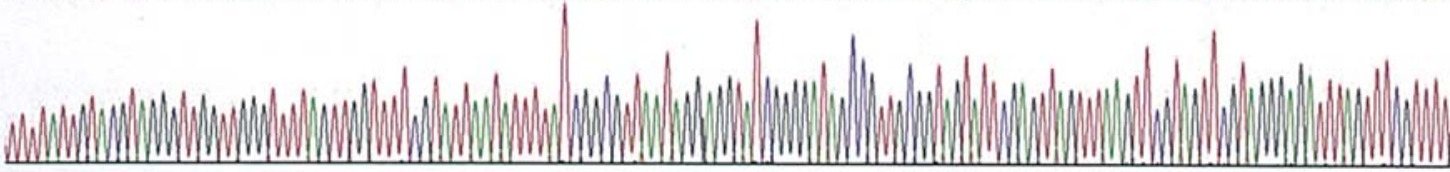

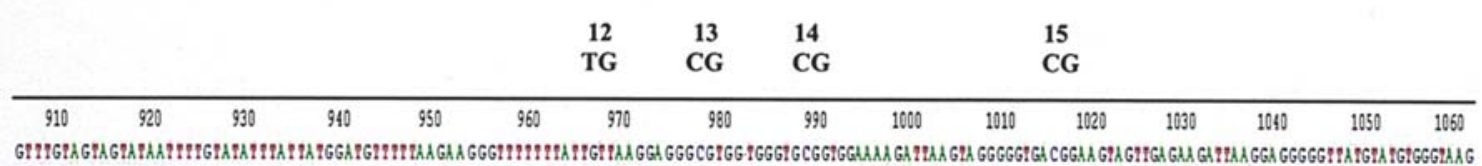

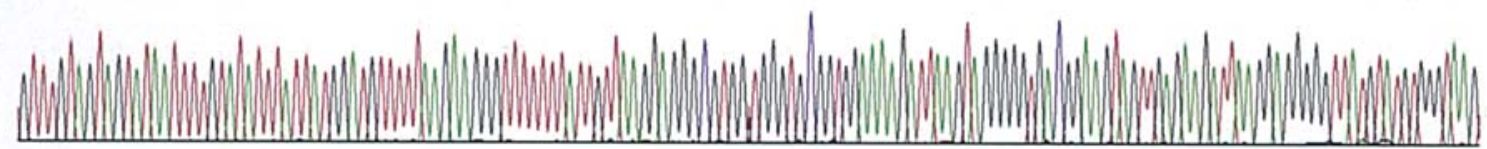

SNCG CpG island in HepG2 cell line after 5-aza-C treatment

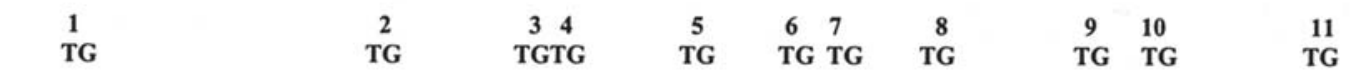

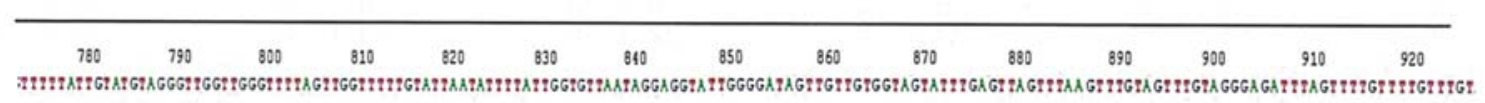

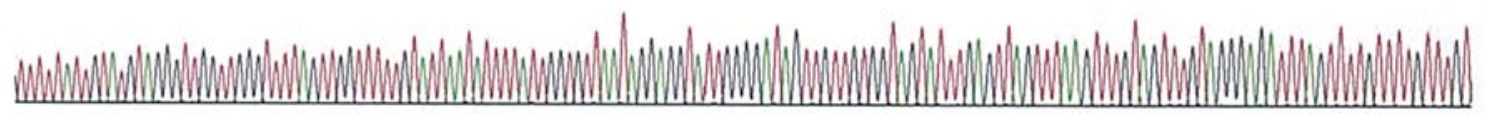

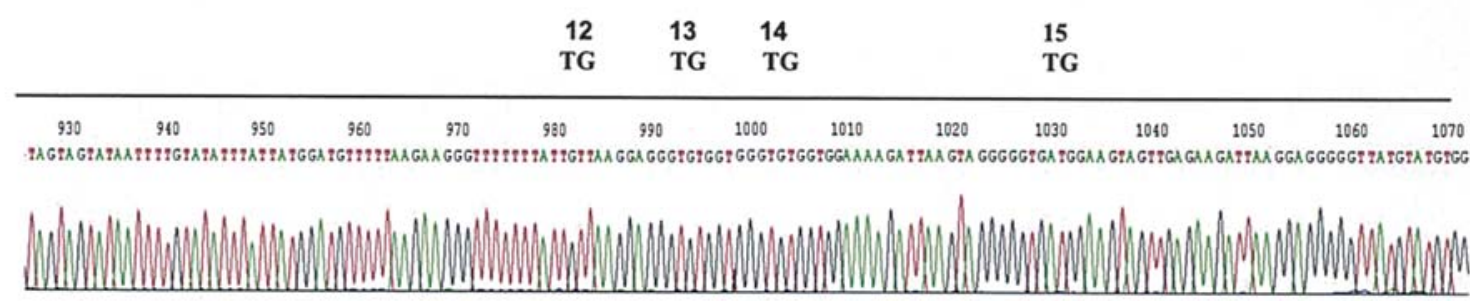

Figure. 5. Changes in the methylation status of the SNCG CpG island and re-expression of SNCG mRNA in HepG2 cells after 5-aza-C treatment. HepG2 cells were treated with 5 and $10 \mu \mathrm{M} 5$-aza-C for 3 and 5 days. The medium was exchanged every day with fresh medium and drug. By the end of treatment, genomic DNA and total RNA were separately isolated from untreated and treated cells. (A) Results of NMSP. (B) Representative results of genomic sequencing of HepG2 DNA samples before and after 5-aza-C treatment. (C) RT-PCR results of SNCG and GAPDH mRNA expression.

island. Significant amounts of unmethylated PCR products appeared after 1-day exposure and became predominant forms in AFB1 and DMN-treated cells after 3-day exposure. We further examined SNCG mRNA expression in AFB1 exposed cells. Fig. 7B shows that SNCG mRNA expression is strongly induced by AFB1 in time- and dose-dependent manners. These results provide the first connection between environmental carcinogens and activation of this oncogene in liver cancer.

\section{Discussion}

The biological heterogeneity and multiple etiologies of HCC result in an incomplete understanding of the key molecular changes that lead to HCC development. Obtaining a clear picture of the most prominent and relevant molecular abnormalities accumulating during $\mathrm{HCC}$ development is fundamental to developing effective treatment options (3). The development of HCC is a complex multistep process. In addition to genetic 

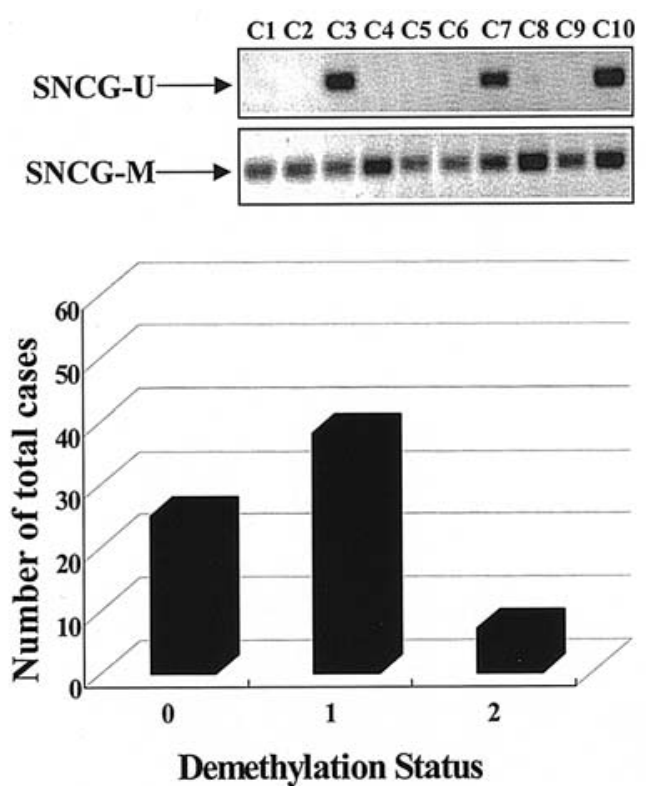

Figure 6. Determination of SNCG CpG island methylation status in cirrhotic liver samples by NMSP. NMSP was used to assess the methylation status of SNCG CpG islands in cirrhotic liver samples. Representative results of 10 samples are shown (upper panel). SNCG-U, unmethylated PCR product; SNCG-M, methylated PCR product. The DNA sample showing only the unmethylated band is scored as 2, the DNA sample showing both unmethylated and methylated is scored as 1, the DNA sample without an unmethylated band is designated a score of 0 . The bar graph summarizes the number of patient samples that are fully demethylated, partially demethylated, or remaining methylated.

changes such as p53 mutations, epigenetic changes including hypermethylation to inactivate tumor suppressor genes, key cell cycle regulators and hypomethylation of oncogenes may also play critical roles in the onset and progression of liver cancer. In this study, we provide strong clinical evidence that suggests a promoting role of the proto oncogene, SNCG, in HCC development.

The CpG island of the SNCG gene is fully methylated in normal liver tissue to keep it transcriptionally silent (19). Utilizing the highly sensitive and specific method of nested MSP, we demonstrate that nearly all liver tumors lost this epigenetic control and contained the demethylated gene. Importantly, our studies also reveal that $64.3 \%$ (45/70) of non-
A

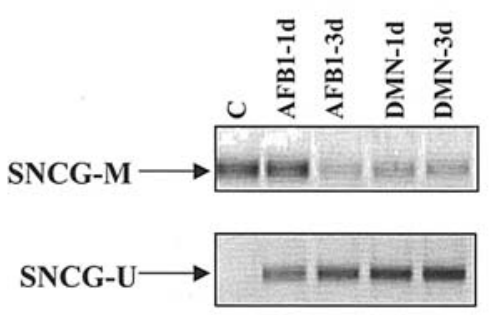

B

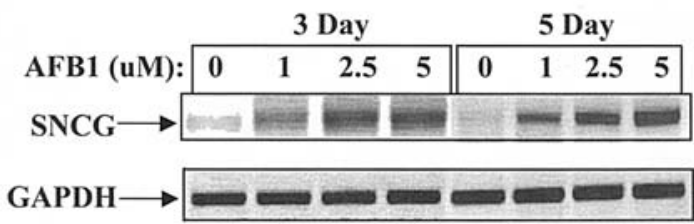

Figure 7. Induction of DNA demethylation of SNCG by genotoxin AFB1 and DMN. In A, HepG2 cells were exposed to AFB1 at a dose of $1 \mu \mathrm{M}$ and to DMN at a dose of $100 \mu \mathrm{M}$ for 1 or 3 days. Genomic DNA was isolated from control and treated cells and subjected to NMSP analysis. In B, AFB1 was added to HepG2 cells at indicated doses for 3 or 5 days and total RNA was isolated by the end of treatment and was subjected to RT-PCR analysis of SNCG and GAPDH mRNA expression.

malignant cirrhotic liver samples contained the demethylated gene, although to lesser extents than liver tumors in which the $\mathrm{CpG}$ island is mostly completely demethylated. The abnormal demethylation of SNCG was mostly found in malignant solid tumors. Liver cirrhosis represents the first example of demethylation of the SNCG gene in non-malignant diseases. Considering the close association of liver cirrhosis with HCC, it is likely that demethylation of the SNCG CpG island is an early event of genetic abnormality occurring in premalignant stages of the disease.

In our study, through examination of 70 tumor samples with different stages of liver cancer, a clear stage-specific expression pattern of SNCG in liver cancer has been manifested. SNCG is expressed in less than $10 \%$ of stage I liver tumors and its expression increases markedly with disease progression. Eighty-three percent of stage III liver cancer expresses SNCG, and more strikingly all stage IV metastatic patients express SNCG in their primary tumors. These results provide strong

Table III. Expression of SNCG in HBV- or HCV-positive human liver tumor tissue.

SNCG protein expression

\begin{tabular}{lccccc} 
Patient cohort & $\begin{array}{c}\text { Hepatitis virus antigen in } \\
\text { tissue (sAg, cAg, HCV) }\end{array}$ & No. & Positive & Negative & \% positive \\
\hline HBV $(+)$ & + & 40 & 26 & 14 & 65 \\
Cohort & - & 30 & 20 & 10 & 66.7 \\
HCV $(+)$ & + & 12 & 7 & 5 & 58.3 \\
Cohort & - & 58 & 39 & 19 & 67.2 \\
\hline
\end{tabular}

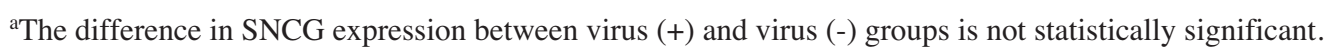


clinical evidence that supports a positive role of SNCG in promoting disease progression and tumor metastasis.

While, thus far, abnormal demethylation of the SNCG CpG island in malignant cancerous cells has been reported in a wide range of human cancers $(18-20,33)$, the cellular, genetic, or environmental factors causing the loss of this important epigenetic control of SNCG gene expression in cancer cells were totally unknown. Identification of such factors would have important implications in cancer prevention. In this study, for the first time, we demonstrate that AFB1 and DMN, two prominent hepatic carcinogens (34), are strong inducers of SNCG expression by causing demethylation of the $\mathrm{CpG}$ island. Thus, hepatic carcinogen exposures may represent one underlying mechanism for the aberrant expression of SNCG in liver cirrhosis and liver cancer.

In conclusion, our studies have firmly demonstrated that epigenetic alteration of the SNCG gene by specific demethylation of the $\mathrm{CpG}$ island is primarily responsible for the abnormal expression of this neuronal protein in liver cancer and in liver cirrhosis. The examination of changes in methylation status of SNCG in cirrhotic livers could be applied in early detection of HCC. Furthermore, SNCG expression in primary liver tumors could be used as a novel biomarker for distant metastasis of liver cancer.

\section{Acknowledgements}

We thank Dr Kelvin Lee (VA Palo Alto HCS) for his help in statistical data analysis. This study was supported by the Department of Veterans Affairs (Office of Research and Development, Medical Research Service and by grants (BC010046 \& BC033154) from the United States Army Medical Research and Material Command.

\section{References}

1. Parkin DM, Pisani P and Ferlay J: Estimates of the worldwide incidence of 25 major cancers in 1990. Int J Cancer 80: 827-841, 1999.

2. Tischoff I, Markwarth A, Witzigmann H, et al: Allele loss and epigenetic inactivation of 3 p21.3 in malignant liver tumors. Int J Cancer 115: 684-689, 2005.

3. Thomas MB and Zhu AX: Hepatocellular carcinoma: the need for progress. J Clin Oncol 23: 2892-2899, 2005.

4. Borlak J, Meier T, Halter R, Spanel R and Spanel-Borowski K: Epidermal growth factor-induced hepatocellular carcinoma: gene expression profiles in precursor lesions, early stage and solitary tumors. Oncogene 24: 1809-1819, 2005.

5. Fausto N: Growth factors in liver development, regeneration and carcinogenesis. Prog Growth Factor Res 3: 219-234, 1991.

6. Ito Y, Takeda T, Sakon M, et al: Expression and clinical significance of erb-B receptor family in hepatocellular carcinoma. Br J Cancer 84: 1377-1383, 2001.

7. Masumoto A, Arao S and Otsuki M: Role of beta 1 integrins in adhesion and invasion of hepatocellular carcinoma cells. Hepatology 29: 68-74, 1999.

8. Yamamoto H, Itoh F, Sakamoto H, et al: Association of reduced cell adhesion regulator messenger RNA expression with tumor progression in human hepatocellular carcinoma. Int J Cancer 74: 251-254, 1997.

9. Mckillop IH, Schmidt CM, Cahill PA, et al: Altered expression of mitogen-activated protein kinases in a rat model of experimental hepatocarcinoma. Hepatology 26: 1484-1491, 1997.

10. Kazachkov Y, Khaoustov V, Yoffe B, et al: p53 abnormalities in hepatocellular carcinoma from United States patients: analysis of all 11 exons. Carcinogenesis 17: 2207-2212, 1996.

11. Liew CT, Li HM, Lo KW, et al: Frequent allelic loss on chromosome 9 in hepatocarcinoma. Int J Cancer 81: 319-324, 1999.
12. Park WS, Cho YG, Kim CJ, et al: Hypermethylation of the RUNX3 gene in hepatocellular carcinoma. Exp Mol Med 37: 276-281, 2005.

13. Zhu JD: The altered DNA methylation pattern and its implications in liver cancer. Cell Res 15: 272-280, 2005.

14. Yu J, Zhang HY, Ma ZZ, Lu W, Wang YF and Zhu J: Methylation profiling of twenty four genes and the concordant methylation behaviors of nineteen genes that may contribute to hepatocellular carcinogenesis. Cell Res 13: 319-333, 2003.

15. Lavedan C, Leroy E, Dehejia A, et al: Identification, localization and characterization of the human $\gamma$-synuclein gene. Hum Genet 103: 106-112, 1998.

16. Ninkina N, Alimova-Kost M, Paterson J, et al: Organization, expression and polymorphism of the human persyn gene. Hum Mol Genet 7: 1417-1424, 1998.

17. Ji H, Liu Y, Jia T, et al: Identification of a breast cancer-specific gene, BCSG1, by direct differential cDNA sequencing. Cancer Res 57: 759-764, 1997.

18. Lu A, Gupta A, Li C, et al: Molecular mechanisms for aberrant expression of the human breast cancer specific gene 1 in breast cancer cells: control of transcription by DNA methylation and intronic sequences. Oncogene 20: 5173-5185, 2001.

19. Liu H, Liu W, Wu YW, et al: Loss of epigenetic control of synuclein-gamma gene as a molecular indicator for metastasis in a wide range of human cancers. Cancer Res 65: 7635-7643, 2005.

20. Gupta A, Godwin AK, Vanderveer L, et al: Hypomethylation of the synuclein gamma gene $\mathrm{CpG}$ island promotes its aberrant expression in breast carcinoma and ovarian carcinoma. Cancer Res 63: 664-673, 2003.

21. Bruening W, Giasson B, Klein-Szanto J, et al: Synucleins are expressed in the majority of breast and ovarian carcinomas and in preneoplastic lesions of the ovary. Cancer 88: 2154-2163, 2000.

22. Wu K, Weng Z, Tao Q, et al: Stage-specific expression of breast cancer-specific gene $\gamma$-synuclein. Cancer Epidemiol Biomarkers Prev 12: 920-925, 2003.

23. Liu J, Spence MJ, Zhang YL, et al: Transcriptional suppression of synuclein $\gamma$ (SNCG) expression in human breast cancer cells by the growth inhibitory cytokine oncostatin M. Breast Cancer Res Treat 62: 99-107, 2000.

24. Lu A, Zhang F, Gupta A and Liu J: Blockade of AP1 transactivation abrogated the abnormal expression of the breast cancer specific gene 1 in breast cancer cells. J Biol Chem 277: 31364-31372, 2002.

25. Jiang Y, Liu YE, Lu A, et al: Stimulation of estrogen receptor signaling by gamma synuclein. Cancer Res 63: 3899-3903, 2003.

26. Jia T, Liu Y, Liu J and Shi Y: Stimulation of breast cancer invasion and metastasis by breast cancer-specific synuclein (SNCG). Cancer Res 59: 742-747, 1999.

27. Jiang GF, Liu YA, Goldberg ID and Shi YE: $\gamma$ synuclein, a novel heat-shock protein-associated chaperone, stimulates ligand-dependent estrogen receptor $\alpha$ signaling and mammary tumorigenesis. Cancer Res 64: 4539-4546, 2004.

28. Gupta A, Inaba S, Wong OK, et al: Breast cancer-specific gene 1 interacts with the mitotic checkpoint kinase BubR1. Oncogene 22: 7593-7599, 2003.

29. Pan Z, Bruening W, Giasson BI, et al: $\gamma$-Synuclein promotes cancer cell survival and inhibits stress- and chemotherapy druginduced apoptosis by modulating MAPK pathways. J Biol Chem 277: 35050-35060, 2002.

30. Inaba S, Li C, Shi YE, et al: Synuclein gamma inhibits the mitotic checkpoint function and promotes chromosomal instability of breast cancer cells. Breast Cancer Res Treat 94: 25-35, 2005

31. Esteller M, Sparks A, Toyota M, et al: Analysis of adenomatous polyposis coli promoter hypermethylation in human cancer. Cancer Res 60: 4366-4371, 2000.

32. Harris AJ, Dial SL and Casciano DA: Comparison of basal gene expression profiles and effects of hepatocarcinogens on gene expression in cultured primary human hepatocytes and HepG2 cells. Mutat Res 549: 79-99, 2004.

33. Yanagawa N, Tamura G, Honda T, et al: Demethylation of the synuclein $\gamma$ gene $\mathrm{CpG}$ island in primary gastric cancers and gastric cancer cell lines. Clin Cancer Res 10: 2447-2451, 2004.

34. Sharma RA and Farmer PB: Biological relevance of adduct detection to the chemoprevention of cancer. Clin Cancer Res 10: 4901-4912, 2004. 